\title{
Paeonol Protects Rat Heart by Improving Regional Blood Perfusion during No-Reflow
}

\author{
Lina $\mathrm{Ma}^{1,2 \dagger}$, Chia-Chen Chuang ${ }^{3,4 \dagger}$, Weiliang Weng $^{2}$, Le Zhao ${ }^{2}$, Yongqiu Zheng ${ }^{27}$, \\ Jinyan Zhang ${ }^{2 f}$ and Li Zuo ${ }^{3,4 *}$
}

\begin{abstract}
${ }^{1}$ Graduate School, Beijing University of Chinese Medicine, Beijing, China, ${ }^{2}$ Institute of Basic Medical Sciences, Xiyuan Hospital, China Academy of Chinese Medical Sciences, Beijing, China, ${ }^{3}$ Radiologic Sciences and Respiratory Therapy Division, School of Health and Rehabilitation Sciences, The Ohio State University College of Medicine, Columbus, OH, USA, ${ }^{4}$ Interdisciplinary Biophysics Graduate Program, The Ohio State University, Columbus, OH, USA
\end{abstract}

OPEN ACCESS

Edited by:

Leonardo Alexandre Peyré-Tartaruga, Universidade Federal do Rio Grande do Sul, Brazil

Reviewed by:

Davide Susta,

Dublin City University, Ireland Rochelle Rocha Costa

Federal University of Rio Grande do

Sul, Brazil

Flavia Alessandra Guarnier, Universidade Estadual de Londrina

Brazil

*Correspondence:

Li Zuo

zuo.4@osu.edu

${ }^{\dagger}$ These authors have contributed equally to this work and co-first

authors.

${ }^{*}$ These authors have contributed equally to this work

Specialty section:

This article was submitted to Integrative Physiology, a section of the journal Frontiers in Physiology

Received: 28 March 2016 Accepted: 28 June 2016 Published: 21 July 2016

Citation: Ma L, Chuang C-C, Weng W, Zhao L, Zheng Y, Zhang J and Zuo L (2016) Paeonol Protects Rat Heart by Improving Regional Blood Perfusion during No-Reflow.

Front. Physiol. 7:298. doi: 10.3389/fphys.2016.00298
No-reflow phenomenon, defined as inadequate perfusion of myocardium without evident artery obstruction, occurs at a high incidence after coronary revascularization. The mechanisms underlying no-reflow is only partially understood. It is commonly caused by the swelling of endothelial cells, neutrophil accumulation, and vasoconstriction, which are all related to acute inflammation. Persistent no-reflow can lead to hospitalization and mortality. However, an effective preventive intervention has not yet been established. We have previously found that paeonol, an active extraction from the root of Paeonia suffruticosa, can benefit the heart function by inhibiting tissue damage after ischemia, reducing inflammation, and inducing vasodilatation. To further investigate the potential cardioprotective action of paeonol on no-reflow, healthy male Wistar rats were randomly divided into four groups: sham, ischemia-reperfusion (l/R) injury (left anterior descending coronary artery was ligated for $4 \mathrm{~h}$ followed by reperfusion for $8 \mathrm{~h}$ ), and I/R injury pretreated with paeonol at two different doses. Real-time myocardial contrast echocardiography was used to monitor regional blood perfusion and cardiac functions. Our data indicated that paeonol treatment significantly reduces myocardial infarct area and no-reflow area $(n=8 ; p<0.05)$. Regional myocardial perfusion (A. $\beta$ ) and cardiac functions such as ejection fraction, stroke volume, and fractional shortening were elevated by paeonol $(n=8 ; p<0.05)$. Paeonol also lowered the serum levels of lactate dehydrogenase, creatine kinase, cardiac troponin $\mathrm{T}$, and $\mathrm{C}$-reactive protein, as indices of myocardial injury. Paeonol exerts beneficial effects on attenuating I/R-associated no-reflow injuries, and may be considered as a potential preventive treatment for cardiac diseases or post-coronary revascularization in which no-reflow often occurs.

Keywords: paeonol, myocardial injury, no-reflow, infarct size, rat

\section{INTRODUCTION}

Ischemic heart disease is a major cause of morbidity and mortality worldwide (Zuo et al., 2009, 2013; Zhu and Zuo, 2013; Sivaraman and Yellon, 2014). Coronary revascularization, including thrombolysis, percutaneous coronary intervention (PCI), and bypass grafting, is recognized as the most effective therapeutic strategy for coronary artery diseases (Niccoli et al., 2009; Serruys et al., 2009). However, a subset of patients continues to suffer from severe myocardial ischemia 
caused by factors such as myocardial interstitial edema, microvascular spasm, endothelial cell injury/swelling, neutrophil accumulation, micro-thromboembolism, and vasoconstriction related to acute inflammation (Rezkalla and Kloner, 2002; Kaul, 2014; Shao et al., 2014). The persistence of these symptoms, termed no-reflow phenomena, can cause insufficient regional blood perfusion, left ventricular (LV) dysfunction, and even cardiac death (Kawano et al., 2005; Chan et al., 2012; Zeng et al., 2012). Several attempts have been made to resolve no-reflow phenomenon with novel interventions associated with Western medicine, yet no ideal strategy has been established (Ramjane et al., 2008; Salinas et al., 2012).

Approaches that use traditional Chinese medicinal plants and their active ingredients, such as Tongxinluo capsule, have been emerging due to their multi-targeted pharmacological effects during no-reflow treatment (Wu et al., 2006; Liu et al., 2011). Other Chinese medicines, such as paeonol, a main constituent extracted from the root of Paeonia suffruticosa, is regarded as a traditional Chinese herb that belongs to the Ranunculaceae family. Studies have observed that paeonol exhibits a wide range of biological effects, including anti-inflammatory, immune regulatory, anti-tumor, and anti-oxidative effects (Sun et al., 2008; Jin et al., 2016). Our previous studies on rat models have demonstrated that paeonol is therapeutically beneficial for myocardial infarction by inhibiting tissue damage after ischemia and inducing vasodilatation in the mesenteric artery in vivo (Li Y.K. et al., 2010; Zhang et al., 2013). Other studies showed that paeonol can reduce myocardial damage by preventing apoptosis in vivo (Nizamutdinova et al., 2008). However, our understanding of paeonol as a cardioprotective agent against myocardial ischemia/reperfusion (I/R)-associated no-reflow phenomenon, is still largely unknown. Therefore, the current study particularly aims to investigate the effect of paeonol on no-reflow and regional blood perfusion during myocardial I/R in rat models. We hypothesize that paeonol exerts cardioprotective effects by attenuating I/R-associated no-reflow injuries and therefore may be considered as a potential preventive treatment for cardiac diseases or post-coronary revascularization when no-reflow occurs.

\section{MATERIALS AND METHODS}

\section{Experimental Animals and Reagents}

Healthy male Wistar rats (180-220 g, average age of 8 week) were procured from Beijing Si Beifu Laboratory Animal Technology Co., Ltd. All animal experiments were performed in accordance with the standards established by the Institutional Animal Care and Use Committee of Institute of Basic Medical Sciences of Xiyuan Hospital, and were approved by the Beijing Local Ethics Review Committee, Beijing University Laboratory Animal Center (Animal Permit \#: SCXK Beijing 2011-0004). The rats were randomly divided into four groups: (1) sham group, thoracotomy without left anterior descending coronary artery (LAD) occlusion or paeonol pretreatment; (2) I/R group, LAD occlusion (ischemia) for $4 \mathrm{~h}$ followed by reperfusion for $8 \mathrm{~h}$; (3) Paeonol $(100 \mathrm{mg} / \mathrm{kg})+\mathrm{I} / \mathrm{R}$ group, oral administration of $100 \mathrm{mg} / \mathrm{kg}$ paeonol $(1 \mathrm{~mL} / \mathrm{kg})$ for 7 days using a intragastric tube prior to I/R procedure; (4) Paeonol (200 mg/kg) + I/R group, oral administration of $200 \mathrm{mg} / \mathrm{kg}$ paeonol $(1 \mathrm{~mL} / \mathrm{kg})$ for 7 days using a intragastric tube prior to $\mathrm{I} / \mathrm{R}$ procedure. In addition, rats in the sham and I/R groups received a dosage of dimethyl sulfoxide (DMSO, Beijing Solarbio Science \& Technology Co., Ltd., China) equal to that with which the paeonol was dissolved in for the other two groups. DMSO was also administered intragastrically for 7 consecutive days. A minimum of eight rats were assigned to each group (Zhang et al., 2014). An ischemia group without reperfusion is not included since our present study mainly focuses on the effect of paeonol on the cardiac injuries after reperfusion, which is closely related to the real-world situation of no-reflow after coronary revascularization. However, future studies may include a group subjected only to $4 \mathrm{~h}$ of ischemia to differentiate, in terms of damage to the cardiac function, which was due to the ischemia and which was due to the no-reflow.

Previous literature reported a dose-dependent (30, 50, $100 \mathrm{mg} / \mathrm{kg}$ ) effect of paeonol in reducing inflammation (Chou, 2003). In our myocardial I/R models, $100 \mathrm{mg} / \mathrm{kg}$ paeonol was used as low-dose and $200 \mathrm{mg} / \mathrm{kg}$ as high-dose to ensure a range of dosage effectiveness. In addition, to our best knowledge, the duration of drug action for paeonol has not yet been fully investigated. Taken into account of this, we designed a 7-day administration period of pretreatment to ensure a sufficient time for the onset of paeonol. Future studies will focus on the drug action as well as the dosage effectiveness of paeonol.

Paeonol (purity $>95 \%$ ) was obtained from Ningbo Dekang Biologic Product Co., Ltd. (Ningbo, China). Nitro-blue tetrazolium chloride (NBT) and chloral hydrate were purchased from Sinopharm Chemical Reagent Co., Ltd. (Beijing, China). Thioflavin S was purchased from Sigma (St. Louis, MO, USA). Lactate dehydrogenase (LDH), creatine kinase (CK), cardiac troponin $\mathrm{T}$ (cTnT), and C-reactive protein (CRP) assay kits were purchased from BioSino Bio-technology and Science Inc. (Beijing, China). All of the chemicals were dissolved in physiological saline, except for paeonol, which was dissolved in DMSO.

\section{Establishment of Myocardial I/R Rat Model}

The myocardial I/R model was established previously by Michael et al. (1995). Briefly, rats were anesthetized via an intraperitoneal (IP) injection of $3.5 \%$ chloral hydrate $(1 \mathrm{~mL} / \mathrm{kg})$ and placed in a supine position with all paws secured on the operating table. The left thorax was opened to expose the heart. The LAD coronary artery was ligated and the chest was closed by a hemostat for 4 consecutive hours until the reperfusion took place (Zhang et al., 2014). Although in rodent heart, 30-60 min ischemia is normally sufficient to induce myocardial infarction, ischemia for $4 \mathrm{~h}$ in our setting would lead to a more severe risk region in order to fully evaluate the therapeutic efficacy of paeonol during no-reflow, as described previously (Zhang et al., 2014). Compared to models with relatively shorter ischemia periods, no-reflow area and perfusion area were more clearly identified in our unique $4 \mathrm{~h}$ ischemic model, and the heart remained viable after this prolonged ischemia caused by LAD ligation since other coronary arteries were still able to partially support the blood flow to the left ventricle (Zhang et al., 2014). 
Following the removal of LAD ligation during another chest opening for reperfusion, the chest was re-sutured and the analysis was performed $8 \mathrm{~h}$ later from the start of reperfusion. In the sham group, the rat underwent the same chest surgery in the absence of LAD ligation. The body temperature of the rats was maintained at all time. The chest opening time during LAD occlusion and ligation removal was kept at minimum by experienced surgeons. Successful induction of acute myocardial I/R model was confirmed by visual inspection of color alteration in the LV and ST segment-characterized electrocardiogram.

\section{Cardiac Function by Echocardiography}

Following $8 \mathrm{~h}$ of reperfusion, rats were adequately sedated by $3 \%$ isoflurane (inhaled). Ultrasound gel was placed on the precordial region of the shaved chest wall, and ultrasound bio-microscopy (Vevo 770, Visual-Sonics Inc., Toronto, ON, Canada) with a $17.5-\mathrm{MHz}$ transducer was used to visualize the left ventricle. The LV wall thickness, chamber diameter, and pressure were measured from the two-dimensional directed images of the LV (M-mode; short axis below papillary muscle), as previously described (Samuel et al., 2008). LV function was assessed by measuring LV end-systolic and end-diastolic volumes, ejection fraction (EF), stroke volume (SV), and fractional shortening (FS). All measurements were recorded from at least three consecutive cardiac cycles and the mean values were reported.

\section{Myocardial Contrast Echocardiography (MCE) Analysis}

Real-time MCE was used to capture MCE sequences during cardiac cycles and quantitatively analyze the extent of myocardial blood flow. Regions of interest (ROIs) were positioned at the anterior wall of LV to measure the non-perfused areas in our models. Constant infusion of microbubble contrast agent $(0.8 \mathrm{ml}$ of $11.8 \mathrm{mg} / \mathrm{ml}$ per mouse) was performed via tail vein injection. The total imaging acquisition lasted for $\sim 12.5 \mathrm{~s}$. The signal intensity increased immediately following the injection of contrast agents. The displayed pattern of the curve included an ascending slope followed by a plateau, indicating an increase followed by stabilization of the detectable intensity from contrast agents. The time-dependent perfusion in the heart can be described with a mathematical model, which can be solved using nonlinear regression analysis. The exponential curve of time versus intensity was then graphed based on previous studies (Raher et al., 2007; Sun et al., 2011).

Signal variation is modeled as $y=\mathrm{A}\left(1-e^{\beta \mathrm{t}}\right)$, where $y$ is the contrast agent replenishment data at any given time. A (plateau intensity) and $\beta$ (rate constant of signal intensity rise) represent microvascular cross-sectional area and blood velocity, respectively. These values are automatically generated by MCE software. On the other hand, the calculated product A. $\beta$ provides an estimation of myocardial blood flow (Wei et al., 1998; Raher et al., 2007).

\section{Measurement of Serum Parameters}

After the completion of cardiac function assessment and MCE analysis, thioflavin $\mathrm{S}(6 \%, 1 \mathrm{~mL} / \mathrm{kg})$ was injected into the femoral vein of all rats for the subsequent examination of the infarct area. Blood was immediately collected from the abdominal aorta and centrifuged at $1050 \times g$ for $2 \times 10 \mathrm{~min}$. The serum was analyzed using assay kits to detect the activities of $\mathrm{LDH}, \mathrm{CK}$, cTnT, and CRP. All procedures were performed according to

A

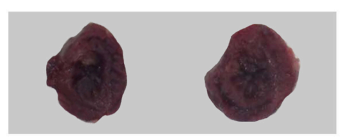

Sham

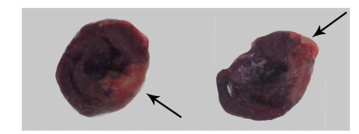

Paeonol

$(100 \mathrm{mg} / \mathrm{kg})+\mathrm{I} / \mathrm{R}$

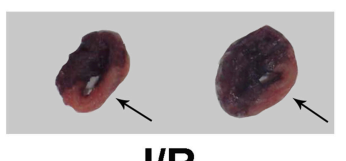

I/R

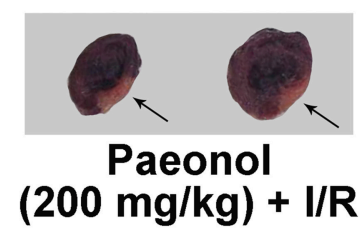

B

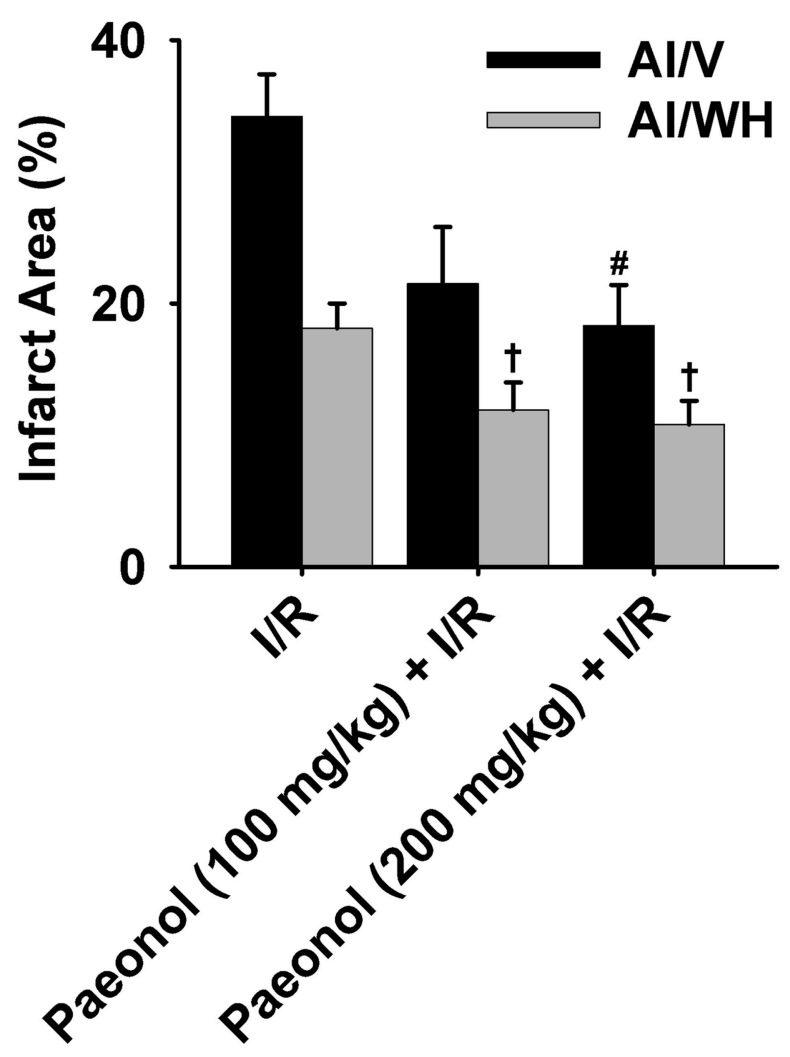

FIGURE 1 | Effects of paeonol on myocardial infarct area in ischemia/reperfusion (I/R) rats. (A) Representative images of the infarct area for all four groups [Sham, $1 / \mathrm{R}$, paeonol $(100 \mathrm{mg} / \mathrm{kg})+\mathrm{I} / \mathrm{R}$, paeonol $(200 \mathrm{mg} / \mathrm{kg})+\mathrm{l} / \mathrm{R}]$, with the arrows showing nitro-blue tetrazolium chloride (NBT)-negative infarct areas. (B) The percentage of the infarct area to the ventricle size $(\mathrm{Al} / \mathrm{M})$ and to the whole heart section $(\mathrm{Al} / \mathrm{WH})$ in the three groups. I/R group $(n=9)$, paeonol $(100 \mathrm{mg} / \mathrm{kg})+\mathrm{l} / \mathrm{R}$ group $(n=8)$, paeonol $(200 \mathrm{mg} / \mathrm{kg})+\mathrm{I} / \mathrm{R}$ group $(n=8)$. The data are expressed as mean $\pm \mathrm{SE}$. ${ }^{\#} p<0.01$, compared with $\mathrm{l} / \mathrm{R}$ group of the ventricle size. ${ }^{\dagger} p<0.05$, compared with I/R group of the whole heart. 
the manufacturer's protocol (Goldberg et al., 2006; Xia et al., 2014).

\section{Myocardial Staining for No-Reflow and Infarct Area Evaluation}

Rats were sacrificed immediately by removing the whole heart. The heart was transected into five even slices parallel to the atrioventricular groove. The slices were photographed under ultraviolet light (UV, $365 \mathrm{~nm}$ ) to identify the region of no-reflow. By thioflavin S staining, areas that were perfused with blood were fluorescent, whereas areas that were not perfused appeared dark (Shao et al., 2013). The slices were then incubated in $0.2 \%$ NBT for $3 \mathrm{~min}$ in room temperature, immersed in formalin, and photographed again. Infarct area was unstained by NBT and the non-infarct area was stained dark brown (Kloner et al., 1974). The no-reflow and infarct areas were measured by a multimedia color pathological image analytical system (MPIAS-500, Beijing Kong Hai Science and Technology Development Co., Ltd., China). Data of no-reflow area were determined as the percentage of the no-reflow area to the ventricle size (AN/V) or the no-reflow area to the whole heart section $(\mathrm{AN} / \mathrm{WH})$. Data of infarct area were determined as the percentage of the infarct area to the ventricle size (AI/V) or the infarct area to the whole heart section $(\mathrm{AI} / \mathrm{WH}$; Shao et al., 2013).

\section{Statistical Analysis}

Data were analyzed using SPSS 17.0 software (SPSS Inc., Chicago, IL, USA) and the values are expressed as mean \pm standard error (SE). A normality test was performed on all presented data and the differences between each group were assessed by nonparametric Mann-Whitney test. The results were considered significant at $p<0.05$.

\section{RESULTS}

\section{Effects of Paeonol on Myocardial Infarct Area}

Initial in vivo experiment of paeonol was performed in rat models of myocardial I/R induced by LAD ligation. Different doses of paeonol (100 and $200 \mathrm{mg} / \mathrm{kg}$ ) were used during the pretreatment of two experimental groups. Myocardial injury was evident by the quantitative measurement of acute myocardial infarct sizes. The $200 \mathrm{mg} / \mathrm{kg}$ paeonol $+\mathrm{I} / \mathrm{R}$ group $[\mathrm{AI} / \mathrm{V}(\%): 18.3 \pm 3.1, p<0.01$; $\mathrm{AI} / \mathrm{WH}(\%): 10.8 \pm 1.8, p<0.05]$ and $100 \mathrm{mg} / \mathrm{kg}$ paeonol $+\mathrm{I} / \mathrm{R}$ group [AI/WH (\%): $11.9 \pm 2.1, p<0.05]$ exhibited significant reductions in the infarct area compared with the I/R group [AI/V (\%): $34.2 \pm 3.2 ; \mathrm{AI} / \mathrm{WH}(\%): 18.1 \pm 1.9$ ] (Figure 1, Datasheet 2). However, the comparison between the $100 \mathrm{mg} / \mathrm{kg}$ paeonol + $\mathrm{I} / \mathrm{R}$ group and the $200 \mathrm{mg} / \mathrm{kg}$ paeonol $+\mathrm{I} / \mathrm{R}$ group showed no significant difference in the infarct area reduction $(\mathrm{AI} / \mathrm{V}, p>$ 0.05 ; $\mathrm{AI} / \mathrm{WH}, p>0.05$ ).

\section{Effects of Paeonol on No-Reflow Area}

The $200 \mathrm{mg} / \mathrm{kg}$ paeonol + I/R group [AN/V (\%): $7.6 \pm 2.2, p<$ $0.01]$ and $100 \mathrm{mg} / \mathrm{kg}$ paeonol $+\mathrm{I} / \mathrm{R}$ group [AN/V (\%): $9.4 \pm 2.8$, $p<0.05]$ both showed lesser extents of no-reflow area in the ventricles compared with the I/R group [AN/V (\%): $18.2 \pm 2.9$ ].

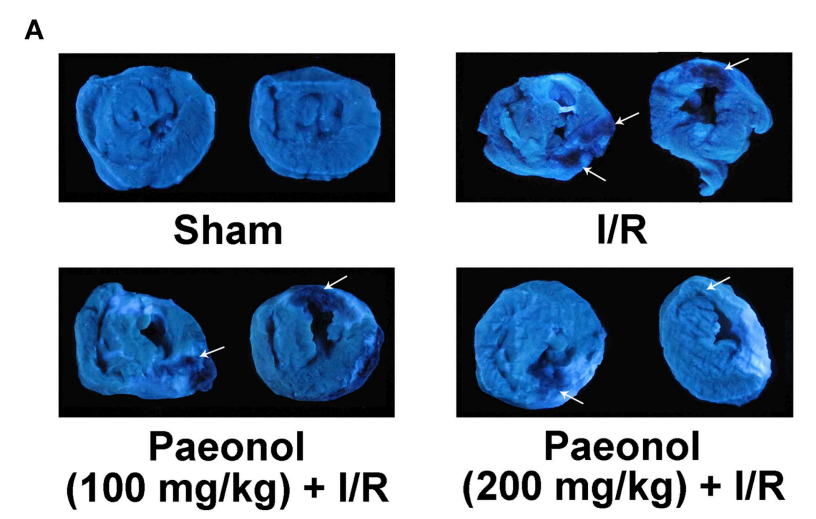

B

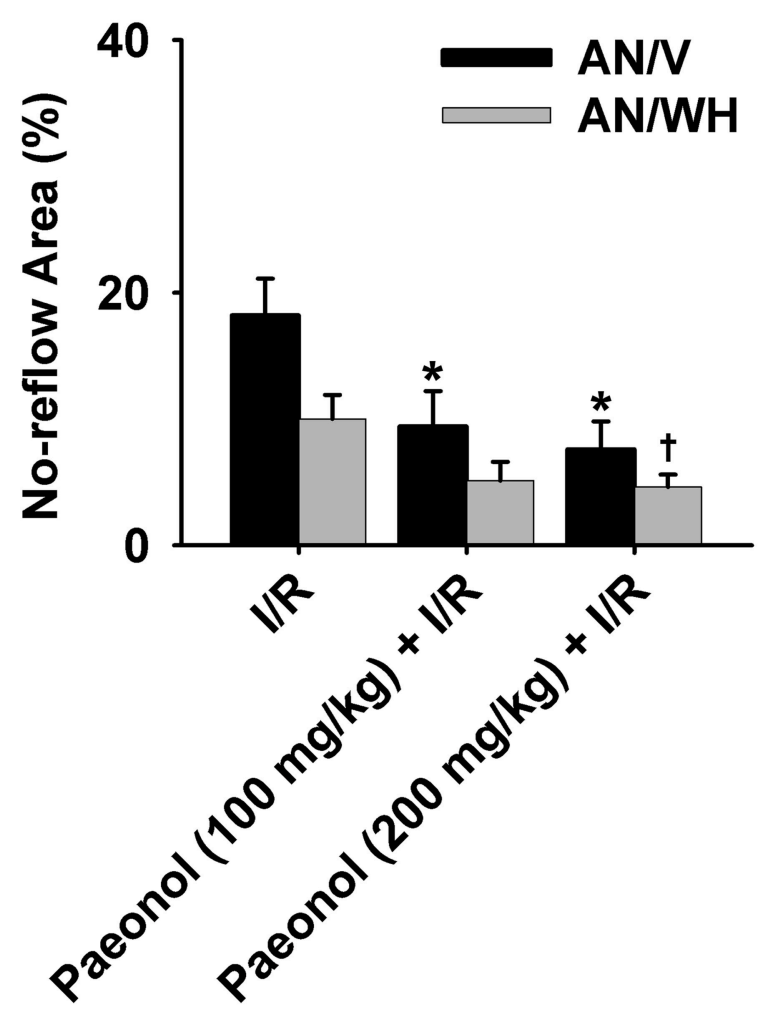

FIGURE 2 | Effects of paeonol on no-reflow area in myocardial ischemia/reperfusion (I/R) rats. (A) Representative images of the no-reflow area for all four groups [Sham, I/R, paeonol $(100 \mathrm{mg} / \mathrm{kg})+\mathrm{I} / \mathrm{R}$, paeonol $(200 \mathrm{mg} / \mathrm{kg})+\mathrm{l} / \mathrm{R}]$, with the arrows showing no-reflow areas. (B) The percentage of the no-reflow area to the ventricle size (AN/N) and to the whole heart section (AN/WH) in the three groups. I/R group $(n=9)$, paeonol $(100 \mathrm{mg} / \mathrm{kg})+\mathrm{l} / \mathrm{R}$ group $(n=8)$, paeonol $(200 \mathrm{mg} / \mathrm{kg})+\mathrm{l} / \mathrm{R}$ group $(n=8)$. The data are expressed as mean \pm SE. ${ }^{*} p<0.05$, compared with $\mathrm{I} / \mathrm{R}$ group of the ventricle size. ${ }^{\dagger} p<0.05$, compared with $\mathrm{l} / \mathrm{R}$ group of the whole heart.

In particular, the $200 \mathrm{mg} / \mathrm{kg}$ paeonol $+\mathrm{I} / \mathrm{R}$ group experienced markedly alleviated no-reflow in the whole heart [AN/WH (\%): $4.6 \pm 1.0, p<0.05$ ] compared with the I/R group [AN/WH (\%): $10.0 \pm 1.9]$ (Figure 2, Datasheet 4). Furthermore, no significant difference in no-reflow area was found between the two dosages of paeonol (AN/V, $p>0.05$; AN/WH, $p>0.05$ ). 


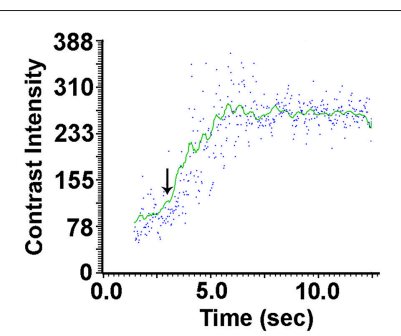

Sham

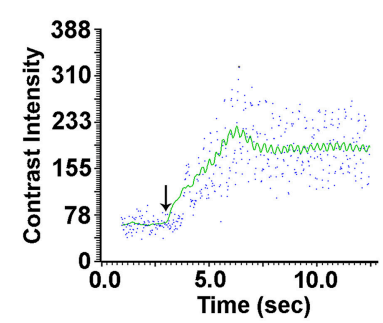

Paeonol

$(100 \mathrm{mg} / \mathrm{kg})+\mathrm{I} / \mathrm{R}$

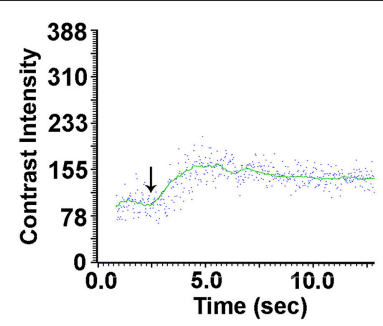

I/R

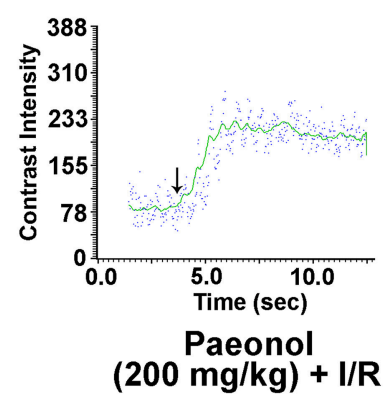

FIGURE 3 | Effect of paeonol on regional myocardial blood flow. Representative images of regional myocardial blood flow of the four groups. Sham group $(n=9)$, I/R group $(n=9)$, paeonol $(100 \mathrm{mg} / \mathrm{kg})+\mathrm{I} / \mathrm{R}$ group $(n=8)$, paeonol $(200 \mathrm{mg} / \mathrm{kg})+\mathrm{l} / \mathrm{R}$ group $(n=8)$. The arrows indicate the time point for the contrast agent injection.

\section{Effects of Paeonol on Regional Myocardial Blood Flow}

The quantitation of paeonol's effect on the regional myocardial blood flow was assessed by MCE (Figure 3 and Table 1). The results showed that $\beta(0.28 \pm 0.03 / \mathrm{s}, p<0.05)$, A (102.21 \pm $11.69 \mathrm{~d} \beta, p<0.01)$ and $A \cdot \beta(26.76 \pm 2.39 \mathrm{~d} \beta / \mathrm{s}, p<0.001)$ were significantly decreased in the I/R group compared with the sham group $(0.42 \pm 0.05 / \mathrm{s}, 193.33 \pm 13.34 \mathrm{~d} \beta, 86.32 \pm 18.65 \mathrm{~d} \beta / \mathrm{s})$, respectively. In the paeonol-pretreated groups, $\beta(200 \mathrm{mg} / \mathrm{kg}$ paeonol group: $0.43 \pm 0.05 / \mathrm{s}, p<0.05 ; 100 \mathrm{mg} / \mathrm{kg}$ paeonol group: $0.55 \pm 0.11 / \mathrm{s}, p<0.01)$, A $(200 \mathrm{mg} / \mathrm{kg}$ paeonol group: $173.79 \pm 15.99 \mathrm{~d} \beta, p<0.01 ; 100 \mathrm{mg} / \mathrm{kg}$ paeonol group: 183.83 $\pm 17.01 \mathrm{~d} \beta, p<0.01)$ and $\mathrm{A} \cdot \beta(200 \mathrm{mg} / \mathrm{kg}$ paeonol group: 70.01 $\pm 7.06 \mathrm{~d} \beta / \mathrm{s}, p<0.001 ; 100 \mathrm{mg} / \mathrm{kg}$ paeonol group: $87.32 \pm 14.82$ $\mathrm{d} \beta / \mathrm{s}, p<0.001$ ), were significantly increased as compared to that in the I/R group (Table 1, Datasheet 3). However, no significant dose-dependent difference in MCE results was observed between two doses of paeonol $(100 \mathrm{mg} / \mathrm{kg}$ vs. $200 \mathrm{mg} / \mathrm{kg} ; \beta, p>0.05 ; \mathrm{A}$, $p>0.05 ; \mathrm{A} \cdot \beta, p>0.05)$.

\section{Effects of Paeonol on Cardiac Function in Myocardial I/R Rats}

We also examined whether paeonol alters cardiac functional parameters in myocardial $\mathrm{I} / \mathrm{R}$ rats using echocardiographic recording (Figure 4). The LV anterior wall systolic and diastolic thicknesses (LVAW d/s) were significantly improved in the $200 \mathrm{mg} / \mathrm{kg}$ paeonol $+\mathrm{I} / \mathrm{R}$ group $(n=8 ; p<0.05)$ and the $100 \mathrm{mg} / \mathrm{kg}$ paeonol $+\mathrm{I} / \mathrm{R}$ group (LVAW d; $n=8$; $p<0.01)$ compared with that in the I/R group. However,
TABLE 1 | Quantitative MCE analysis of regional myocardial blood flow.

\begin{tabular}{llll}
\hline & \multicolumn{1}{c}{$\mathbf{A}(\mathbf{d B})$} & $\boldsymbol{\beta}\left(\mathbf{s}^{-\mathbf{1}}\right)$ & $\mathbf{A} \cdot \boldsymbol{\beta}(\mathbf{d B} / \mathbf{s})$ \\
\hline Groups & & & \\
Sham $(n=9)$ & $193.33 \pm 13.34$ & $0.42 \pm 0.05$ & $86.32 \pm 18.65$ \\
I/R $(n=9)$ & $102.21 \pm 11.69^{\Delta}$ & $0.28 \pm 0.03^{\ddagger}$ & $26.76 \pm 2.39^{\S}$ \\
Paeonol $(100 \mathrm{mg} / \mathrm{kg})+$ & $183.83 \pm 17.01^{\#}$ & $0.55 \pm 0.11^{\#}$ & $87.32 \pm 14.82^{\dagger}$ \\
l/R $(n=8)$ & & & \\
Paeonol $(200 \mathrm{mg} / \mathrm{kg})+$ & $173.79 \pm 15.99^{\#}$ & $0.43 \pm 0.05^{\star}$ & $70.01 \pm 7.06^{\dagger}$ \\
I/R $(n=8)$ & & & \\
\hline
\end{tabular}

$A$, plateau; $\beta$, rise rate of the signal intensity; $A \cdot \beta$, regional myocardial perfusion. Values are mean $\pm S E$.

${ }^{*} p<0.05,{ }^{*} p<0.01,{ }^{\dagger} p<0.001$ vs. I/R group.

${ }^{\ddagger} p<0.05,{ }^{\Delta} p<0.01,{ }^{\S} p<0.001$ vs. sham group.
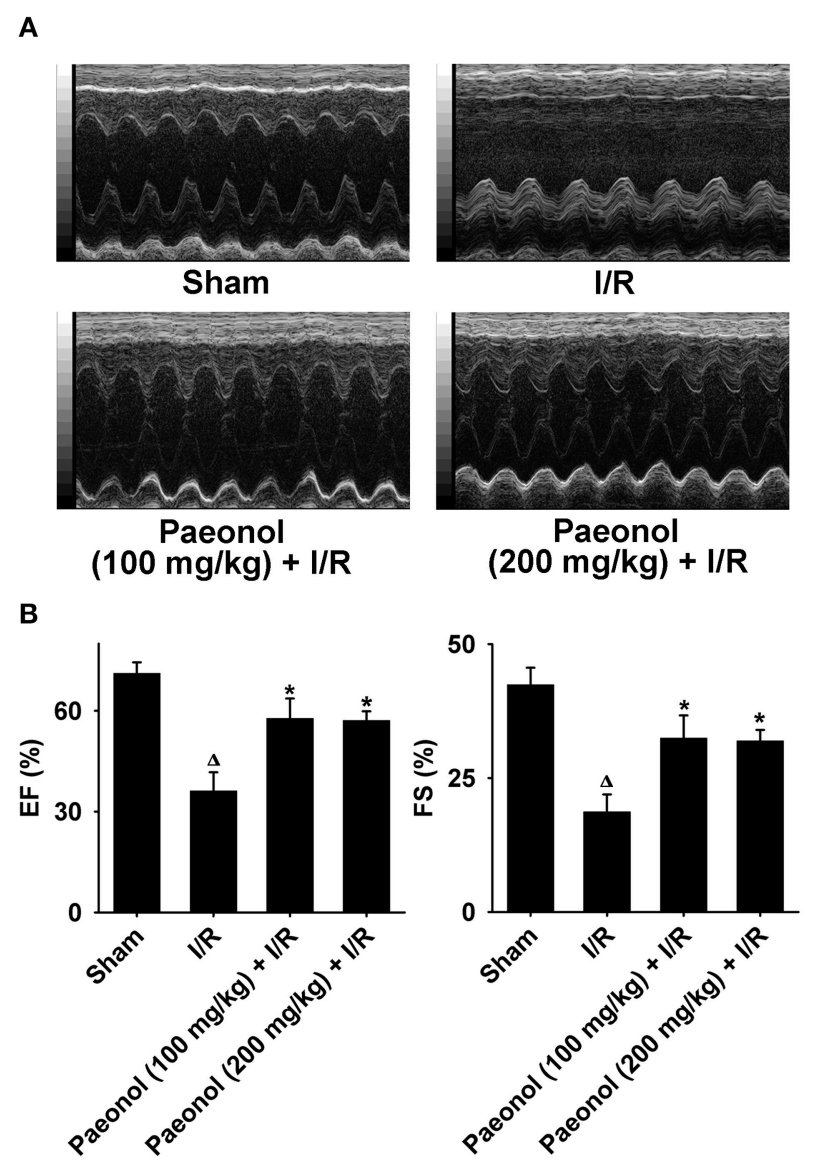

FIGURE 4 | Effect of paeonol on cardiac function evaluated by echocardiography. (A) Representative echocardiographic images of the four groups after the surgical procedure. Each image shown is 1-s duration of the entire echocardiograph. (B) Percentage of ejection fraction (EF) and fractional shortening (FS) affected by paeonol. Sham group ( $n=9)$, I/R group $(n=9)$, paeonol $(100 \mathrm{mg} / \mathrm{kg})+\mathrm{I} / \mathrm{R}$ group $(n=8)$, paeonol $(200 \mathrm{mg} / \mathrm{kg})+\mathrm{I} / \mathrm{R}$ group $(n=8)$. ${ }^{*} p<0.05$ compared with $\mathrm{I} / \mathrm{R}$ group. ${ }^{\Delta} p<0.001$ compared with sham group.

no marked differences in the LV posterior wall diastolic and systolic thickness (LVPW d/s) were observed. The LV internal diameter in systole (LVID s) was reduced only in the $200 \mathrm{mg} / \mathrm{kg}$ 
TABLE 2 | Echocardiographic analysis of cardiac function in four groups.

\begin{tabular}{|c|c|c|c|c|}
\hline & Sham & I/R & Paeonol $(100 \mathrm{mg} / \mathrm{kg})+\mathrm{I} / \mathrm{R}$ & Paeonol $(200 \mathrm{mg} / \mathrm{kg})+\mathrm{I} / \mathrm{R}$ \\
\hline$n$ & 9 & 9 & 8 & 8 \\
\hline LVAW d (mm) & $1.89 \pm 0.07$ & $1.49 \pm 0.08^{\ddagger}$ & $1.86 \pm 0.12^{\#}$ & $1.89 \pm 0.10^{*}$ \\
\hline LVAW s (mm) & $2.75 \pm 0.10$ & $1.63 \pm 0.11^{\Delta}$ & $1.97 \pm 0.10^{*}$ & $2.11 \pm 0.08^{\#}$ \\
\hline LVPW d (mm) & $1.90 \pm 0.08$ & $1.87 \pm 0.09$ & $1.86 \pm 0.11$ & $2.02 \pm 0.07$ \\
\hline LVPW s (mm) & $2.67 \pm 0.11$ & $2.21 \pm 0.23$ & $2.58 \pm 0.20$ & $2.84 \pm 0.28$ \\
\hline LVID d (mm) & $6.70 \pm 0.15$ & $7.64 \pm 0.29^{\dagger}$ & $7.34 \pm 0.36$ & $7.03 \pm 0.14$ \\
\hline LVID s (mm) & $3.91 \pm 0.27$ & $6.30 \pm 0.41^{\Delta}$ & $5.25 \pm 0.50$ & $4.84 \pm 0.19^{\#}$ \\
\hline LW d $(\mu \mathrm{l})$ & $236.10 \pm 10.77$ & $316.18 \pm 26.06^{\dagger}$ & $253.31 \pm 23.84$ & $257.92 \pm 13.69$ \\
\hline LV $s(\mu l)$ & $70.32 \pm 9.71$ & $208.88 \pm 30.55^{\ddagger}$ & $116.07 \pm 26.36^{\star}$ & $110.55 \pm 10.68^{\#}$ \\
\hline EF (\%) & $71.13 \pm 3.25$ & $36.17 \pm 5.53^{\Delta}$ & $57.73 \pm 5.91^{\star}$ & $57.09 \pm 2.73^{*}$ \\
\hline FS (\%) & $42.41 \pm 3.17$ & $18.68 \pm 3.25^{\Delta}$ & $32.44 \pm 4.24^{*}$ & $31.92 \pm 2.08^{*}$ \\
\hline SV $(\mu \mathrm{l})$ & $165.78 \pm 4.76$ & $107.30 \pm 12.89^{\Delta}$ & $137.24 \pm 5.76$ & $147.37 \pm 7.71^{*}$ \\
\hline
\end{tabular}

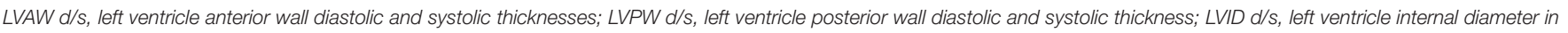
diastole and systole; $L W \mathrm{~d} / \mathrm{s}$, left ventricle volume in diastole and systole; EF, ejection fraction; FS, fractional shortening; SV, stroke volume.

Values are mean $\pm S E$.

${ }^{*} p<0.05,{ }^{*} p<0.01 \mathrm{vs}$. I/R group.

${ }^{\dagger} p<0.05,{ }^{\ddagger} p<0.01,{ }^{\Delta} p<0.001$ vs. sham group.

paeonol-pretreated + I/R group $(n=8 ; p<0.01)$, and LVID in diastole (LVID d) in both paeonol-pretreated groups showed no significant difference when compared to I/R group. The LV volume in systole (LVV s) was significantly decreased $(n=8$; $p<0.05)$ in both $200 \mathrm{mg} / \mathrm{kg}$ paeonol $+\mathrm{I} / \mathrm{R}$ group and $100 \mathrm{mg} / \mathrm{kg}$ paeonol $+\mathrm{I} / \mathrm{R}$ group, whereas LVV in diastole (LVV d) expressed no marked difference (Table 2). Along with elevated EF and FS in both paeonol-pretreated groups ( $n=8$; $p<0.05$; Table 2), these results showed improved cardiac function with paeonol treatment. Interestingly, greater SV was observed only in the $200 \mathrm{mg} / \mathrm{kg}$ paeonol $+\mathrm{I} / \mathrm{R}$ group $(n=8 ; p<0.05)$, yet no significant difference was seen in the $100 \mathrm{mg} / \mathrm{kg}$ paeonol $+\mathrm{I} / \mathrm{R}$ group (Table 2, Datasheet 1).

\section{Effects of Paeonol on Serum LDH, CK, cTnT, and CRP Levels in Myocardial I/R Rats}

$\mathrm{LDH}, \mathrm{CK}, \mathrm{cTnT}$, and CRP concentrations were all elevated in the I/R group $[\mathrm{LDH}(\mathrm{U} / \mathrm{L}): 1128.67 \pm 124.80, p<0.05$; CK (U/L): $686.28 \pm 46.25, p<0.05 ;$ cTnT $(\mu \mathrm{g} / \mathrm{L}): 181.94$ \pm 4.72, $p<0.001 ; \mathrm{CRP}(\mathrm{ng} / \mathrm{L}): 1570.96 \pm 60.31, p<$ 0.001] compared with the sham group [LDH (U/L): 607.11 \pm 114.35 ; CK $(\mathrm{U} / \mathrm{L}): 468.56 \pm 50.93$; $\mathrm{cTnT}(\mu \mathrm{g} / \mathrm{L}): 24.89 \pm$ 1.16; CRP (ng/L): $1024.18 \pm 55.45]$. In the paeonol-pretreated groups, a marked reduction in LDH concentration was observed in $100 \mathrm{mg} / \mathrm{kg}$ paeonol $+\mathrm{I} / \mathrm{R}$ group [LDH (U/L): $730.28 \pm$ 167.25]; yet $200 \mathrm{mg} / \mathrm{kg}$ paeonol $+\mathrm{I} / \mathrm{R}$ group [LDH (U/L): 774.00 \pm 69.97; CK (U/L): $486.99 \pm 61.83$; cTnT ( $\mu \mathrm{g} / \mathrm{L}): 167.03 \pm$ 2.63; CRP (ng/L): $1396.79 \pm 42.88$ ] significantly declined all four biomarker measurements compared with the I/R group $(n=9 ; p<0.05$, Figures 5A-D, Datasheet 5). Moreover, the comparison between the two paeonol-pretreated groups showed no significant difference in LDH, CK, cTnT, and CRP levels $(p>0.05)$.

\section{DISCUSSION}

No-reflow phenomenon emerges when blood fails to reperfuse an ischemic area after coronary revascularization (Shao et al., 2013). When no-reflow occurs, pharmacological treatments including intracoronary verapamil and adenosine, as well as mechanical therapies (e.g., aspiration thrombectomy) can be used to partially relieve microvasculature obstruction and improve blood flow in the heart. However, the development of a more efficient treatment, especially the prevention of no-reflow, still remains extremely challenging due to the complexity of this phenomenon (Ramjane et al., 2008; Berg and Buhari, 2012). As one of the active compounds in P. suffruticosa, paeonol has been shown to exert beneficial effects, such as vascular dilation and arrhythmia improvements (Li Y.J. et al., 2010; Li et al., 2012). Our findings of the effect of paeonol on regional blood perfusion in the present study are consistent with the aforementioned reports. In particular, our results further indicated that pretreatment with paeonol reduced no-reflow area in conjunction with marked decreased release of serum CK and $\mathrm{LDH}$, as well as a slight decline of cTnT and CRP. Our study is the first to show that paeonol pretreatment has pharmacologically protective effects on the no-reflow phenomenon. This result, consistent with our hypothesis, provides evidence that using paeonol may be a preventive strategy for no-reflow injuries in cardiac diseases or post-coronary revascularization, warranting pre-clinical testing in other models.

Myocardial infarction and no-reflow are the major manifestations of reperfusion injury (Kloner et al., 2012). Our data showed that paeonol significantly minimized the no-reflow area. Consequently, the same trend was observed in the infarct area, indicating that the extent of no-reflow was likely correlated with the extent of infarction, consistent with literature (Coggins et al., 2001). Furthermore, we observed a reduction in the release of $\mathrm{LDH}, \mathrm{CK}, \mathrm{cTnT}$, and CRP, all 
A

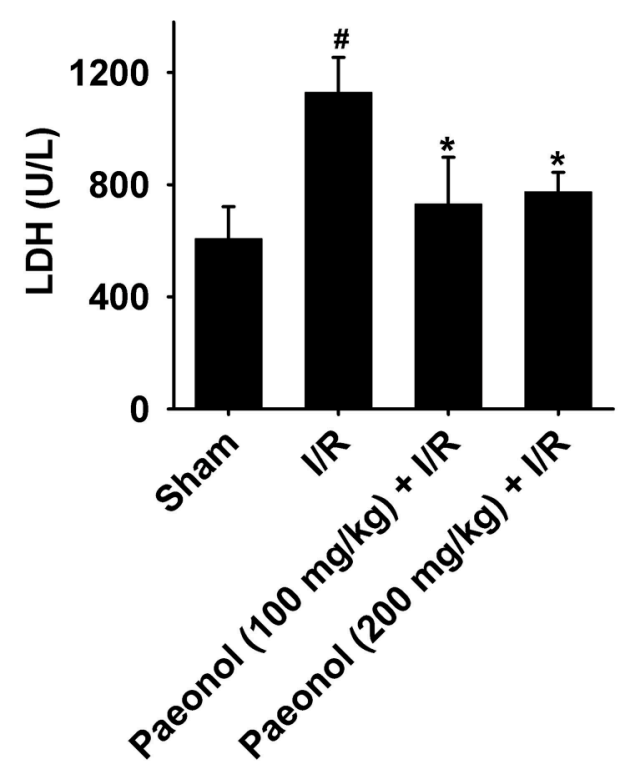

C

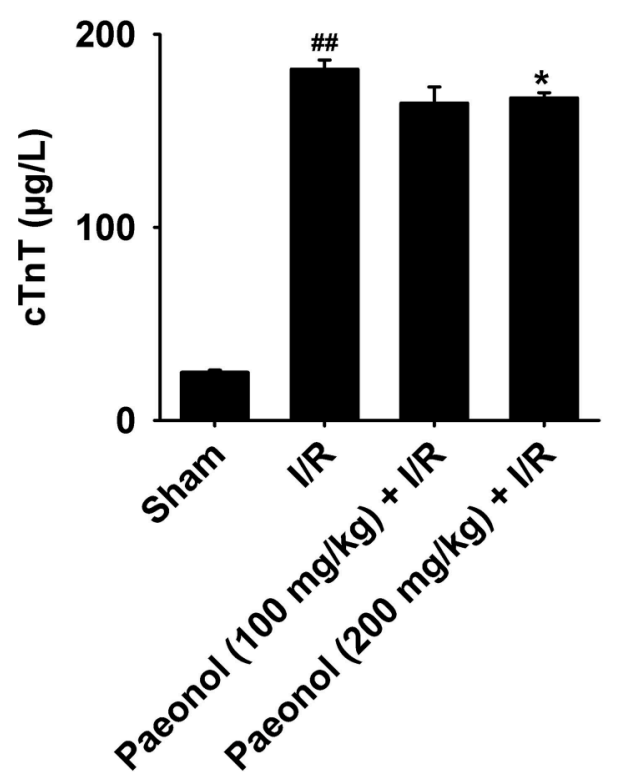

B

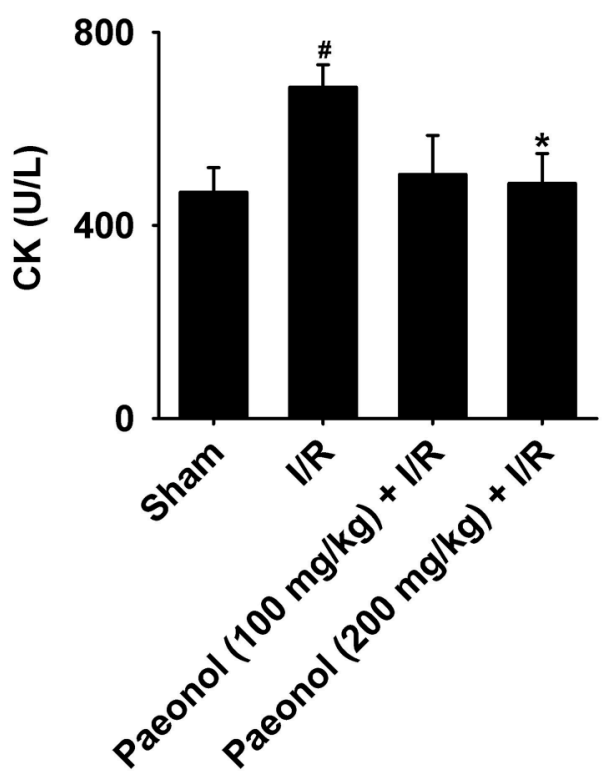

D

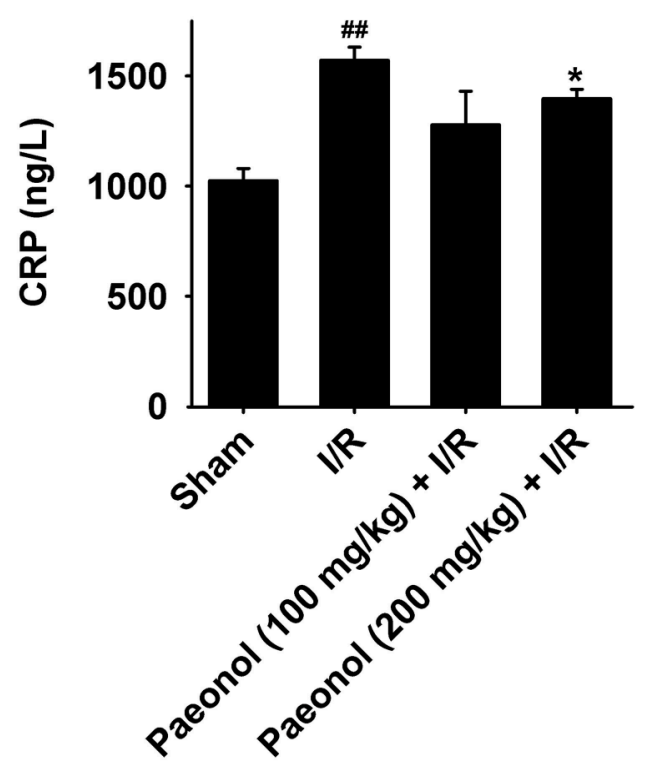

FIGURE 5 | Effects of paeonol on the levels of serum biomarkers, lactate dehydrogenase (LDH; A), creatine kinase (CK; B), cardiac troponin T (cTnT; C), and C-reactive protein (CRP; D), in the four groups. Sham group $(n=9), \mathrm{l} / \mathrm{R}$ group $(n=9)$, paeonol $(100 \mathrm{mg} / \mathrm{kg})+\mathrm{l} / \mathrm{R}$ group $(n=8)$, paeonol $(200 \mathrm{mg} / \mathrm{kg})+$ I/R group $(n=8)$. The data are expressed as mean \pm SE. ${ }^{*} p<0.05$, compared with $\mathrm{I} / \mathrm{R}$ group; $\# p<0.05$, \#\# $p<0.001$, compared with sham group.

of which are indicators of potential myocardial injuries in $200 \mathrm{mg} / \mathrm{kg}$ paeonol-pretreated rat group, although no significant dose-dependent difference is observed between the two doses of paeonol. LDH and CK are important markers of cellular necrosis (Xia et al., 2014). Elevated CK and cTnT levels, which are often associated with systemic inflammation, have been detected in angina patients undergoing PCI (Goldberg et al., 2006). Thus, our results suggested that the paeonol treatment may alleviate myocardial injury in rat models at molecular levels.
Real-time MCE is a promising echocardiography technology that is clinically applied for the noninvasive evaluation of myocardial capillary perfusion in patients (Agati et al., 2004). Given that myocardial infarctions are closely related to the insufficient blood supply (Wagner et al., 2002; Thygesen et al., 2007), we examined regional myocardial blood flow by MCE in myocardial $I / R$ rats. We found that paeonol significantly increased the plateau $(A)$, rise rate of the signal intensity $(\beta)$, and regional myocardial perfusion $(A \cdot \beta)$ in both paeonol-pretreated 
groups. These observations showed that paeonol pretreatment reduced myocardial ischemic damage most likely via a potential modulation of microvasculature. In the long run, paeonol may enhance the blood supply of impaired microvessels, thereby impeding myocardial I/R-associated no-reflow. Based on our results, LVAW, EF, FS, and SV were dramatically elevated, whereas LVID s and LVV s were markedly decreased compared with the groups with no paeonol pretreatment, benefitting cardiac performance. However, some cardiac functions including LVID $\mathrm{d}$ and LVV $\mathrm{d}$ showed no significant difference in the paeonol-pretreated groups compared to I/R groups, which is likely due to the use of non-optimal dosage of paeonol and/or insufficient duration of administration in the present study. Notably, no marked difference in certain measured parameters (including infarct area, no-reflow area, regional myocardial blood flow and cardiac function) was found between the two doses of paeonol, indicating the importance of evaluating and determining the dose effectiveness, administration duration, and onset of paeonol.

The upregulation of $\mathrm{Bcl}-2$ family proteins signals cell death under $\mathrm{I} / \mathrm{R}$ stress, but the effect is diminished upon paeonol treatment (Li et al., 2012). Indeed, the most studied pharmacological intervention for no-reflow, verapamil, is a calcium channel blocker that improves coronary blood flow (Berg and Buhari, 2012). Previous studies have shown that paeonol induces vasodilatation of the rat mesenteric arteries by regulating intracellular $\mathrm{Ca}^{2+}$ concentration and improves $\mathrm{Ca}^{2+}$ ATPase activity in the ischemic tissue (Zhang et al., 1997, 2013; Yuan and Jing, 2011). It is possible that paeonol suppresses both $\mathrm{Ca}^{2+}$ overload and hyper-contracture, thus eliciting beneficial effects on cardiac function. However, the exact molecular mechanisms underlying the protective effect of paeonol in no-reflow have not been fully elucidated. Particularly, Li et al. observed an enhanced antioxidant defense in the paeonol-treated rat group, suggesting Nrf2 activation may be involved in the upregulation of paeonol's cardioprotective signaling cascades (Li et al., 2012). Since reactive oxygen species have been shown to play a critical role in almost all biological systems and pathological conditions, including I/R injuries, further research could be focused on the redox mechanism initiated by paeonol (Zuo et al., 2011a,b, 2014, 2015a,b).

We explored the preventive effect of paeonol on noreflow injuries, which commonly occurred after coronary revascularization. Therefore, the administration of paeonol before any potential cardiac attack or at a daily basis may still be a feasible real-world prevention. Limitations of this study may include the fact that the exact molecular mechanisms of paeonol's cardioprotective effects associated with multiple cardiac variables are still not fully understood at the current

\section{REFERENCES}

Agati, L., Tonti, G., Pedrizzetti, G., Magri, F., Funaro, S., Madonna, M., et al. (2004). Clinical application of quantitative analysis in real-time MCE. Eur. J. Echocardiogr. 5(Suppl. 2), S17-S23. doi: 10.1016/s1525-2167(04)80004-0 stage of the study. Our experiment has not yet fully identified the optimal dosages for paeonol's protection to the heart. We did not find any significance of infarct area, no-reflow area, and regional myocardial blood flow between the two doses of paeonol, suggesting the necessity for further experiments to determine the dose effectiveness and administration strategies for paeonol. Moreover, since the paeonol treatment does not have any standard pharmacological treatments in our analyzed variables, future efforts may be focused on the optimal dosage effectiveness as well as the duration of drug action of paeonol.

\section{CONCLUSION}

This study has shown the functional cardioprotective properties of paeonol in vivo rat model. Paeonol minimized the sizes of myocardial infarct area and no-reflow area, and improved regional myocardial blood flow and cardiac function. Our results demonstrate that paeonol may be an alternative drug for clinical application in the prevention of myocardial I/R-associated noreflow and other related cardiac diseases.

\section{AUTHOR CONTRIBUTIONS}

Conception and design of research: WW. Performed experiments: LeZ, JZ. Analyzed data: LM, YZ, JZ, CC, LiZ. Interpreted results of experiments: LM, JZ, CC, LiZ. Prepared figures: LM, CC, LiZ. Drafted manuscript: LM, CC, LiZ. Edited and revised manuscript: LiZ. Approved final version of manuscript: LM, CC, WW, LeZ, YZ, JZ, LiZ.

\section{FUNDING}

This study was supported by China Postdoctoral Science Foundation Fund (2012T50202), Beijing Joint Project Special Funds, as well as OSUCOM-HRS fund 013000 (USA).

\section{ACKNOWLEDGMENTS}

The authors thank $\mathrm{Xu}$ Qing for kindly providing echocardiography; Lianda Li, Andrew Graef, Alexander Ziegler, Tingyang Zhou, and Benjamin Pannell for manuscript preparation.

\section{SUPPLEMENTARY MATERIAL}

The Supplementary Material for this article can be found online at: http://journal.frontiersin.org/article/10.3389/fphys. 2016.00298

Berg, R., and Buhari, C. (2012). Treating and preventing no reflow in the cardiac catheterization laboratory. Curr. Cardiol. Rev. 8, 209-214. doi: $10.2174 / 157340312803217148$

Chan, W., Stub, D., Clark, D. J., Ajani, A. E., Andrianopoulos, N., Brennan, A. L., et al. (2012). Usefulness of transient and persistent no reflow to predict 
adverse clinical outcomes following percutaneous coronary intervention. Am. J. Cardiol. 109, 478-485. doi: 10.1016/j.amjcard.2011.09.037

Chou, T. C. (2003). Anti-inflammatory and analgesic effects of paeonol in carrageenan-evoked thermal hyperalgesia. Br. J. Pharmacol. 139, 1146-1152. doi: 10.1038/sj.bjp.0705360

Coggins, M. P., Sklenar, J., Le, D. E., Wei, K., Lindner, J. R., and Kaul, S. (2001). Noninvasive prediction of ultimate infarct size at the time of acute coronary occlusion based on the extent and magnitude of collateral-derived myocardial blood flow. Circulation 104, 2471-2477. doi: 10.1161/hc4501.0 98954

Goldberg, A., Gruberg, L., Roguin, A., Petcherski, S., Rimer, D., Markiewicz, W., et al. (2006). Preprocedural C-reactive protein levels predict myocardial necrosis after successful coronary stenting in patients with stable angina. Am. Heart J. 151, 1265-1270. doi: 10.1016/j.ahj.2005.07.006

Jin, X., Wang, J., Xia, Z.-M., Shang, C.-H., Chao, Q.-L., Liu, Y.-R., et al. (2016). Anti-inflammatory and Anti-oxidative activities of paeonol and its metabolites through blocking MAPK/ERK/p38 signaling Pathway. Inflammation 39, 434-446. doi: 10.1007/s10753-015-0265-3

Kaul, S. (2014). The "no reflow" phenomenon following acute myocardial infarction: mechanisms and treatment options. J. Cardiol. 64, 77-85. doi: 10.1016/j.jjcc.2014.03.008

Kawano, H., Hayashida, T., Ohtani, H., Kanda, M., Koide, Y., Baba, T., et al. (2005). Histopathological findings of the no-reflow phenomenon following coronary intervention for acute coronary syndrome. Int. Heart J. 46, 327-332. doi: 10.1536/ihj. 46.327

Kloner, R. A., Ganote, C. E., and Jennings, R. B. (1974). The no-reflow phenomenon after temporary coronary occlusion in the dog. J. Clin. Invest. 54, 1496-1508. doi: 10.1172/JCI107898

Kloner, R. A., Hale, S. L., Dai, W., Gorman, R. C., Shuto, T., Koomalsingh, K. J., et al. (2012). Reduction of ischemia/reperfusion injury with bendavia, a mitochondria-targeting cytoprotective Peptide. J. Am. Heart Assoc. 1:e001644. doi: 10.1161/JAHA.112.001644

Li, H., Xie, Y. H., Yang, Q., Wang, S. W., Zhang, B. L., Wang, J. B., et al. (2012). Cardioprotective effect of paeonol and danshensu combination on isoproterenol-induced myocardial injury in rats. PLoS ONE 7:e48872. doi: 10.1371/journal.pone.0048872

Li, Y. J., Bao, J. X., Xu, J. W., Murad, F., and Bian, K. (2010). Vascular dilation by paeonol-a mechanism study. Vascul. Pharmacol. 53, 169-176. doi: 10.1016/j.vph.2010.07.001

Li, Y. K., Hong, X. H., and Zhang, D. (2010). Protective effect of paeonol, paeoniflorin and their different proportion combinations on acute myocardial infarction in rats [Chinese]. Tradit. Chin. Drug Res. Clin. Pharmacol. 21, 254-256.

Liu, H. X., Wang, S. R., Lei, Y., and Shang, J. J. (2011). Characteristics and advantages of traditional Chinese medicine in the treatment of acute myocardial infarction. J. Tradit. Chin. Med. 31, 269-272. doi: 10.1016/s0254$6272(12) 60002-8$

Michael, L. H., Entman, M. L., Hartley, C. J., Youker, K. A., Zhu, J., Hall, S. R., et al. (1995). Myocardial ischemia and reperfusion: a murine model. Am. J. Physiol. 269, H2147-H2154.

Niccoli, G., Burzotta, F., Galiuto, L., and Crea, F. (2009). Myocardial noreflow in humans. J. Am. Coll. Cardiol. 54, 281-292. doi: 10.1016/j.jacc.2009. 03.054

Nizamutdinova, I. T., Jin, Y. C., Kim, J. S., Yean, M. H., Kang, S. S., Kim, Y. S., et al. (2008). Paeonol and paeoniflorin, the main active principles of Paeonia albiflora, protect the heart from myocardial ischemia/reperfusion injury in rats. Planta Med. 74, 14-18. doi: 10.1055/s-2007-993775

Raher, M. J., Thibault, H., Poh, K. K., Liu, R., Halpern, E. F., Derumeaux, G., et al. (2007). In vivo characterization of murine myocardial perfusion with myocardial contrast echocardiography: validation and application in nitric oxide synthase 3 deficient mice. Circulation 116, 1250-1257. doi: 10.1161/CIRCULATIONAHA.107.707737

Ramjane, K., Han, L., and Jin, C. (2008). The diagnosis and treatment of the no-reflow phenomenon in patients with myocardial infarction undergoing percutaneous coronary intervention. Exp. Clin. Cardiol. 13, 121-128.

Rezkalla, S. H., and Kloner, R. A. (2002). No-reflow phenomenon. Circulation 105, 656-662. doi: 10.1161/hc0502.102867
Salinas, P., Jimenez-Valero, S., Moreno, R., Sanchez-Recalde, A., Galeote, G., Calvo, L., et al. (2012). Update in pharmacological management of coronary noreflow phenomenon. Cardiovasc. Hematol. Agents Med. Chem. 10, 256-264. doi: $10.2174 / 187152512802651024$

Samuel, S. M., Thirunavukkarasu, M., Penumathsa, S. V., Paul, D., and Maulik, N. (2008). Akt/FOXO3a/SIRT1-mediated cardioprotection by $\mathrm{n}$-tyrosol against ischemic stress in rat in vivo model of myocardial infarction: switching gears toward survival and longevity. J. Agric. Food Chem. 56, 9692-9698. doi: $10.1021 /$ jf802050h

Serruys, P. W., Morice, M. C., Kappetein, A. P., Colombo, A., Holmes, D. R., Mack, M. J., et al. (2009). Percutaneous coronary intervention versus coronary-artery bypass grafting for severe coronary artery disease. N. Engl. J. Med. 360, 961-972. doi: 10.1056/NEJMoa0804626

Shao, L., Wu, D., Zhang, P., Li, W., Wang, J., Su, G., et al. (2013). The significance of microthrombosis and fgl2 in no-reflow phenomenon of rats with acute myocardial ischemia/reperfusion. Clin. Appl. Thromb. Hemost. 19, 19-28. doi: $10.1177 / 1076029612437577$

Shao, L., Zhang, Y., Ma, A., Zhang, P., Wu, D., Li, W., et al. (2014). Atorvastatin preconditioning improves the forward blood flow in the no-reflow rats. Fundam. Clin. Pharmacol. 28, 42-52. doi: 10.1111/j.1472-8206.2012.01074.x

Sivaraman, V., and Yellon, D. M. (2014). Pharmacologic therapy that simulates conditioning for cardiac ischemic/reperfusion injury. J. Cardiovasc. Pharmacol. Ther. 19, 83-96. doi: 10.1177/1074248413499973

Sun, F. R., Zhang, M. Q., Jia, X. B., Wang, X. J., Yao, G. H., and Zhang, Y. (2011). Region of interest tracking in real-time myocardial contrast echocardiography. J. Med. Syst. 35, 163-167. doi: 10.1007/s10916-009-9353-y

Sun, G. P., Wang, H., Xu, S. P., Shen, Y. X., Wu, Q., Chen, Z. D., et al. (2008). Anti-tumor effects of paeonol in a HepA-hepatoma bearing mouse model via induction of tumor cell apoptosis and stimulation of IL-2 and TNFalpha production. Eur. J. Pharmacol. 584, 246-252. doi: 10.1016/j.ejphar.2008.0 2.016

Thygesen, K., Alpert, J. S., White, H. D., and Joint, E. S. C., and Joint ESC/ACCF/AHA/WHF Task Force for the Redefinition of Myocardial Infarction. (2007). Universal definition of myocardial infarction. Euro. Heart J. 28, 2525-2538. doi: 10.1093/eurheartj/ehm355

Wagner, K. D., Gmehling, G., Gunther, J., Theres, H., Mydlak, K., Schimke, I., et al. (2002). Time-dependent changes of the susceptibility of cardiac contractile function to hypoxia-reoxygenation after myocardial infarction in rats. Mol. Cell. Biochem. 241, 125-133. doi: 10.1023/A:1020841126171

Wei, K., Jayaweera, A. R., Firoozan, S., Linka, A., Skyba, D. M., and Kaul, S. (1998). Quantification of myocardial blood flow with ultrasound-induced destruction of microbubbles administered as a constant venous infusion. Circulation 97, 473-483. doi: 10.1161/01.CIR.97.5.473

Wu, T., Harrison, R. A., Chen, X. Y., Ni, J., Zhou, L., Qiao, J., et al. (2006). Tongxinluo (Tong xin luo or Tong-xin-luo) capsule for unstable angina pectoris. Cochrane Database Syst. Rev. CD004474. doi: 10.1002/14651858.CD004474.pub2

Xia, A., Xue, Z., Li, Y., Wang, W., Xia, J., Wei, T., et al. (2014). Cardioprotective effect of betulinic acid on myocardial ischemia reperfusion injury in rats. Evid. Based Complement. Alternat. Med. 2014:573745. doi: 10.1155/2014/5 73745

Yuan, S. M., and Jing, H. (2011). Insights into the monomers and single drugs of Chinese herbal medicine on myocardial preservation. Afr. J. Tradit. Complement. Altern. Med. 8, 104-127. doi: 10.4314/ajtcam.v8i2.63195

Zeng, M., Yan, H., Chen, Y., Zhao, H. J., Lv, Y., Liu, C., et al. (2012). Suppression of NF-kappaB reduces myocardial no-reflow. PLoS ONE 7:e47306. doi: 10.1371/journal.pone.0047306

Zhang, G., Yu, Z., and Zhao, H. (1997). Protective effect of paeonol on repeated cerebral ischemia in rats [Chinese]. Zhong Yao Cai 20, 626-628.

Zhang, J. Y., Cao, Y. X., Weng, W. L., Li, Y. K., and Zhao, L. (2013). Paeonol induces vasodilatation in rat mesenteric artery via inhibiting extracellular $\mathrm{Ca}(2)(+)$ influx and intracellular $\mathrm{Ca}(2)(+)$ release. Chin. J. Integr. Med. 19, 510-516. doi: 10.1007/s11655-013-1505-8

Zhang, J. Y., Li, Y. K., Li, F. J., Li, S. C., Zhao, L., Hao, W., et al. (2014). Dynamic changes of myocardial infarction area and no-reflow area and their correlation in rats suffering from myocardial ischemia/reperfusion [Chinese]. Tradit. Chin. Drug Res. Clin. Pharmacol. 25, 227-235. 
Zhu, X., and Zuo, L. (2013). Characterization of oxygen radical formation mechanism at early cardiac ischemia. Cell Death Dis. 4:e787. doi: 10.1038/cddis.2013.313

Zuo, L., Best, T. M., Roberts, W. J., Diaz, P. T., and Wagner, P. D. (2015a). Characterization of reactive oxygen species in diaphragm. Acta Physiol. (Oxf) 213, 700-710. doi: 10.1111/apha.12410

Zuo, L., Chen, Y. R., Reyes, L. A., Lee, H. L., Chen, C. L., Villamena, F. A., et al. (2009). The radical trap 5,5-dimethyl-1-pyrroline $\mathrm{N}$-oxide exerts dosedependent protection against myocardial ischemia-reperfusion injury through preservation of mitochondrial electron transport. J. Pharmacol. Exp. Ther. 329, 515-523. doi: 10.1124/jpet.108.143479

Zuo, L., Diaz, P. T., Chien, M. T., Roberts, W. J., Kishek, J., Best, T. M., et al. (2014). PO2 cycling reduces diaphragm fatigue by attenuating ROS formation. PLoS ONE 9:e109884. doi: 10.1371/journal.pone.0109884

Zuo, L., Nogueira, L., and Hogan, M. C. (2011a). Reactive oxygen species formation during tetanic contractions in single isolated Xenopus myofibers. J. Appl. Physiol. (1985) 111, 898-904. doi: 10.1152/japplphysiol.00398.2011

Zuo, L., Roberts, W. J., Tolomello, R. C., and Goins, A. T. (2013). Ischemic and hypoxic preconditioning protect cardiac muscles via intracellular ROS signaling. Front. Biol. 8, 305-311. doi: 10.1007/s11515-012-1225-Z
Zuo, L., Youtz, D. J., and Wold, L. E. (2011b). Particulate matter exposure exacerbates high glucose-induced cardiomyocyte dysfunction through ROS generation. PLOS ONE 6:e23116. doi: 10.1371/journal.pone.00 23116

Zuo, L., Zhou, T., Pannell, B. K., Ziegler, A. C., and Best, T. M. (2015b). Biological and physiological role of reactive oxygen species-the good, the bad and the ugly. Acta Physiol. (Oxf) 214, 329-348. doi: 10.1111/apha. 12515

Conflict of Interest Statement: The authors declare that the research was conducted in the absence of any commercial or financial relationships that could be construed as a potential conflict of interest.

Copyright (c) 2016 Ma, Chuang, Weng, Zhao, Zheng, Zhang and Zuo. This is an open-access article distributed under the terms of the Creative Commons Attribution License (CC BY). The use, distribution or reproduction in other forums is permitted, provided the original author(s) or licensor are credited and that the original publication in this journal is cited, in accordance with accepted academic practice. No use, distribution or reproduction is permitted which does not comply with these terms. 